\title{
О ВЫЧИСЛЕНИИ ТЕПЛОВОГО ИЗЛУЧЕНИЯ ЗЕМНОЙ АТМОСФЕРЫ В СЛУЧАЕ НЕПОЛНОГО КОМПЛЕКСА ИСХОДНЫХ ДАННЫХ
}

Несмотря на значительное расширение возможностей измерения радиационных потоков с помощью спутников, проблемы совершенствования методики вычисления теплового излучения земной атмосферы не утратили своей актуальности. С одной стороны, известно, что точность измерения тепловой радиации остается все еще невысокой, с другой стороны, в некоторых случаях для интерпретации экспериментальных данных требуются дополнительные теоретические сведения и соответствующие расчетные результаты.

Уточнению вычислений часто препятствует отсутствие или недостоверность той или иной группы исходных параметров, например, содержания и вертикального распределения углекислого газа, озона и аэрозоля в атмосфере, а также неполноценность информации о характеристиках влажности. Иногда вертикальное распределение основных метеорологических характеристик (температуры, давления, влажности) над исследуемым районом приходится строить, располагая лишь наземными данными или данными о самом нижнем слое атмосферы, толщина которого определяется высотой полета самолета (вертолета).

Серьезные недостатки, присущие сетевым аэрологическим измерениям влажности, влекут за собой значительные погрешности в количественных данных об атмосферном водяном паре (см., напр., $\left.\left[{ }^{1-3}\right]\right)$. Надежные данные о содержании влаги в атмосфере обеспечивают, как правило, лишь специальные эксперименты, например, оптические и радиометрические. По-видимому, в будущем окажется весьма перспективным определение вертикального хода атмосферной влаги путем решения обратной задачи - восстановления профиля влажности термическим зондированием атмосферы (с помощью либо спутников, либо наземной аппаратуры). Однако в настоящее время подобные исследования еще не стали источниками массовой и регулярной информации, они требуют дальнейшего развития (см. [ $\left.\left.{ }^{4-8}\right]\right)$.

Безусловно, заслуживают серьезного внимания исследования, целью которых является определение общей массы водяного пара в атмосфере по ее собственному тепловому и радиоизлучению, т. е. решение простейшего вида обратной задачи определения влагосодержания атмосферы (см. $\left.\left[{ }^{3,9-15}\right]\right)$. Такие эксперименты уже проводят в рамках практических климатологических исследований: например, в работе В. В. Плечкова и др. $\left[{ }^{11}\right]$ содержатся данные о широтном ходе общей массы водяного пара в атмосфере над Атлантическим океаном, полученные посредством радиометрических измерений вблизи длины волны 1,35 см. 
По нашему мнению, было бы целесообразно включить определение общего влагосодержания атмосферы радиометрическими методами в программу измерений аэрологических и актинометрических станций. Это позволило бы накопить достоверную информацию о количестве влаги в атмосфере, полезную не только для различных климатологических исследований, но и для долгосрочного прогноза погоды. Надежные данные о полной массе водяного пара в атмосфере могли бы служить косвенной проверкой качества данных о влажности, получаемых путем радиозондирования.

Зависимость точности результатов расчетов теплового излучения атмосферы от погрешностей измерения температуры и влажности рассмотрена автором в $\left[{ }^{16}\right]$ *. Оценки, представленные в настоящей работе, сделаны в несколько ином аспекте: перед нами стояла цель выяснить, какова погрешность расчетов теплового излучения атмосферы при наличии неполного комплекса исходных данных и каковы способы достижения при этом наиболее высокой точности результатов.

Ясно, что относительная роль различных групп исходных параметров при определении значений теплового излучения не одинакова. Здесь имеют значение и спектральный интервал, и высота, для которой рассчитывается излучение, и климатические и сезонные условия. Для разработки методики вычисления тепловой радиации необходимо в первую очередь выяснить, какие характеристики для заданных условий являются определяющими, какие второстепенными и какие достаточно знать лишь ориентировочно.

По-видимому, неполный комплекс исходных данных может удовлетворять расчетам теплового атмосферного излучения для нижней тропосферы, где за формирование последнего в основном ответственна сосредоточенная здесь влага, и потому отсутствие точных данных о других составляющих атмосферы не сказывается ощутимо на результатах расчетов. Для верхней же атмосферы, где роль углекислого газа сравнима с ролью водяного пара $\left[{ }^{16}\right]$ и где уже нельзя игнорировать вклад озона, аэрозоля и т. д., желательно иметь полную и достоверную информацию о распределении всех поглощающих компонентов.

Исследование возможных путей приближенных расчетов излучения атмосферы в.настоящей работе базируется на пяти моделях, заимствованных из $\left[{ }^{17}\right]$ : тропики (I), лето в умеренных широтах (II), зима в умеренных широтах (III), приарктическое лето (IV), приарктическая зима $(\mathrm{V})$. В $\left[{ }^{17}\right]$ для всех этих моделей представлены типичные значения давления, температуры и плотности воздуха, а также абсолютной влажности и концентрации озона в атмосфере на высотах от 0 до $100 \kappa M$.

Чтобы наглядно представить влияние того или иного фактора на результаты расчетов длинноволновой радиации атмосферы, рассмотрим пример определения противоизлучения атмосферы на земной поверхности и восходящего излучения на уровне $3 \kappa м$ графическим методом (см. рис. 1 и 2 ).

На рис. 1, где на вертикальную ось нанесены значения функции пропускания атмосферы $P$, а на горизонтальную - значения излучения абсолютного черного тела $B$, численному значению противоизлучения атмосферы соответствует площадь $A_{0} A_{n} C_{n} C_{i} C_{0}$. Как известно, верхняя граница этой площади $\left(A_{0} C_{0}\right)$ всегда находится при $P=1$, а нижняя $\left(A_{n} C_{n}\right)$ определяется функцией пропускания для всей массы атмосферы, $P=P_{\infty}$. Таким образом, одним из первостепенных факторов, ответ-

Статьи автора, опубликованные до 1975 г., изданы под фамилией Х. Ю. Ний- 
ственных за величину площади $A_{0} A_{n} C_{n} C_{0}$, является функция пропускания $P_{\infty}$, которая, в свою очередь, зависит от общей поглощающей массы атмосферы выше того уровня, для которого рассчитывается излучение. Как отмечалось выше, в нижней тропосфере основными поглощателями являются водяной пар и углекислый газ, следовательно, при вычислении противоизлучения атмосферы на земной поверхности решающую роль играют значения полной массы именно этих компонентов.

Точки $C_{0}, C_{1}, C_{2}, \ldots, C_{n}$ устанавливают по температу-

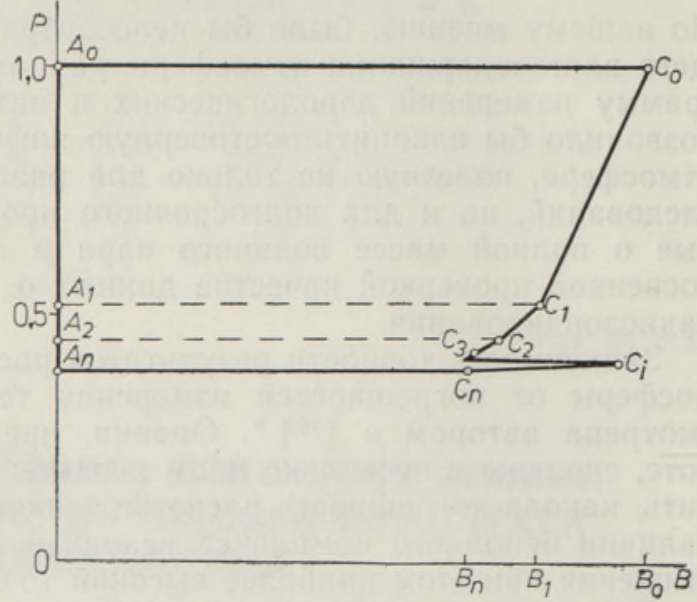

Рис. 1. Схема расчета противонзлучения атмосферы. ре и функции пропускания для высот соответственно $0,1,2, \ldots, n \kappa м$. Таким образом, площадь $A_{0} A_{1} C_{1} C_{0}$ целиком определяется характеристиками атмосферы в слое $0-1 \kappa м$, площадь $A_{0} A_{2} C_{2} C_{0}-$ в слое $0-2 \kappa м$ и т. д. Наклон линий $C_{0} C_{1}, C_{1} C_{2}, C_{2} C_{3}$ и т. д. зависит от градиента температуры в слоях соответственно $0-1,1-2,2-3 \kappa м$ и т. д. Ясно, что отношение площади $A_{0} A_{1} C_{1} C_{0}$ к площади $A_{0} A_{n} C_{n} C_{0}$ характеризует вклад слоя $0-1 \kappa м$ в формирование противоизлучения атмосферы.

Как известно, чем ближе некоторый атмосферный слой находится к уровню, для которого определяется значение радиации, тем бо́льшее влияние он оказывает на формирование потоков излучения. Это проявляется особенно заметно вблизи земной поверхности, где сосредоточена основная масса влаги. Поэтому значительная доля противоизлучения атмосферы происходит от нижних слоев. Произведенные нами ошенки относительного вклада различных слоев атмосферы для моделей $\mathrm{I}-\mathrm{V}$ показали, что слоем 0-1 км обусловливается 85-92\% полного противоизлучения, слоем $0-2 \kappa м-93-97 \%$, слоем $0-3 \kappa м-96-99 \%$. Следовательно, площадь $A_{0} A_{n} C_{n} C_{0}$ определяется в основном атмосферой ниже $3 \kappa м$. и прежде всего слоем $0-1 \kappa м$. Отсюда напрашивается существенный вывод - для расчета противоизлучения атмосферы, кроме значения общего содержания водяного пара и углекислого газа, необходимо иметь надежную и возможно подробную информацию о характеристиках атмосферы именно в пределах нижних слоев (до высот порядка $1-3$ км). Подробные данные о вертикальных распределениях температуры, влажности и других метеорологических параметров для вышележащих слоев имеют явно второстепенное значение; их можно определить путем экстраполяции на основе среднеклиматических данных. Связанная с этим погрешность. характеризующаяся влиянием неточного пасположения отрезка кривой от $C_{3}$ до $C_{n}$ на величину площади $A_{0} A_{n} C_{n} C_{0}$, по нашим оценкам. ниже $0,1 \%$.

Возникает вопрос, в какой мере приведенные выше оценки применимы к вычислению нисходящей тепловой радиации на некоторой высоте от земной поверхности. Решающую роль, по-видимому, здесь играет влагосодержание атмосферы. Например, для модели V общая

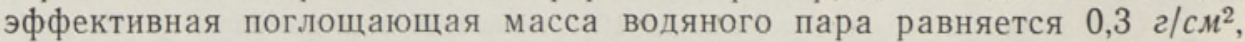
а для модели I такая же масса влаги наблюдается выше 3,5 км (общая 


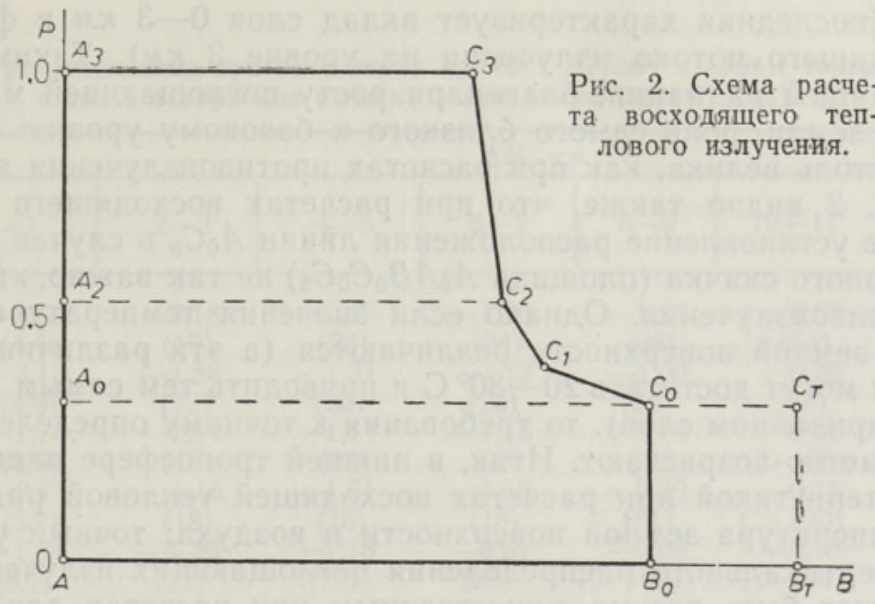

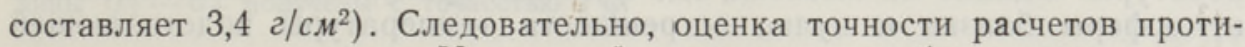
воизлучения для модели $\mathrm{V}$ у земной поверхности в общих чертах должна быть справедлива для уровня $3,5 \kappa$ модели I.

В литературе приводятся многочисленные варианты полуэмпирических формул и методов для расчета противоизлучения и эффективного излучения вблизи земной поверхности на основании лишь наземных метеорологических данных $\left[{ }^{18-21}\right]$. По-видимому, популярность этих методов объясняется именно огромным влиянием приземного слоя атмосферы на значения радиационных потоков, что и обусловливает хорошую корреляцию наземной влажности и температуры с атмосферной радиацией. Однако ясно, что надежные результаты подобных расчетов гарантированы только тогда, когда наземные значения влаги хорошо коррелируют и с общим содержанием водяного пара в атмосфере. Данные различных авторов о выполняемости такой корреляции весьма противоречивы, некоторые исследователи считают ее даже весьма слабой (см., напр., $\left[{ }^{22}\right]$ ).

На рис. 2 показан расчет восходящего излучения земли и атмосферы для уровня $H=3 \kappa м$. В действительности рис. 2 отражает два варианта температурных условий у земной поверхности - площадь $A_{3} A B_{0} C_{0} C_{3}$ соответствует случаю, когда температура почвы равняется температуре воздуха у земли, а площадь $A_{3} A B_{T} C_{T} C_{0} C_{3}-$ случаю, когда имеется температурный скачок (почва перегрета на несколько градусов относительно воздуха). Для простоты предполагается, что излучательная способность подстилающей поверхности равна единице.

Как известно, верхняя и нижняя границы $\left(A_{3} C_{3}\right.$ и $\left.A B_{0}\left(A B_{T}\right)\right)$ этих площадей всегда определяются значениями $P=1$ и 0 соответственно. Однако местоположение линии $A_{0} C_{0}\left(A_{0} C_{T}\right)$ зависит от значения функции пропускания для слоя атмосферы от 0 до $3 \kappa$, т. е. от содержания поглощающих радиацию компонентов в этом слое. Кривая $C_{3} C_{2} C_{1} C_{0}$ строится по данным изменения температуры и поглощающей массы в пределах от 0 до $3 \kappa$, причем ординаты точек $C_{i}$ определяются функцией пропускания, а абсциссы - значениями температуры $\left(C_{3}\right.$ соответствует высоте $3 \kappa м, C_{2}-2 \kappa м$ и т. д.). Произведенные оценки относительного вклада самых близких к базовому уровню (в данном случае 3 км) слоев атмосферы в формирование интегрального восходящего излучения показали, что для моделей $\mathrm{I}-\mathrm{V}$ вклад слоя $2-3 \kappa м$ составляет примерно $40-60 \%$ площади $A_{3} A B_{0} C_{0} C_{3}$ и около $75 \%$ площади 
$A_{3} A_{0} C_{0} C_{3}$ (последняя характеризует вклад слоя $0-3 \kappa м$ в формирование восходящего потока излучения на уровне $3 \kappa м$ ). Таким образом, при восходящей радиации, благодаря росту поглощающей массы в направлении земли, роль самого близкого к базовому уровню слоя атмосферы не столь велика, как при расчетах противоизлучения атмосферы.

Из рис. 2 видно также, что при расчетах восходящего излучения правильное установление расположения линии $A_{0} C_{0}$ в случае отсутствия температурного скачка (площадь $A_{3} A B_{0} C_{0} C_{3}$ ) не так важно, как при расчетах противоизлучения. Однако если значения температуры почвы и воздуха у земной поверхности различаются (а эти различия в жаркие летние дни могут достигать $20-30^{\circ} \mathrm{C}$ и приводить тем самым к ошибкам до $25 \%$ в приземном слое), то требования к точному определению линии $A_{0} C_{0} C_{T}$ заметно возрастают. Итак, в нижней тропосфере наиболее важной характеристикой при расчетах восходящей тепловой радиации является температура земной поверхности и воздуха; точный учет содержания и вертикального распределения поглощающих излучение компонентов может быть весьма существенным при расчетах для свободной атмосферы, особенно в случае большого вертикального градиента температуры. Однако на высотах, где функция пропускания нижележащей атмосферы близка к нулю, погрешности температуры подстилающей поверхности практически уже не влияют на результаты расчетов.

Говоря о разработке оптимальной методики расчета теплового излучения атмосферы, необходимо иметь представление о требуемой точности результатов вычисления величин радиации. Ясно, что степень точности определяется характером и целью исследований. Наиболее острым, однако, остается вопрос о том, как гарантировать высокие результаты расчета радиационных притоков тепла. Поскольку в большинстве случаев этот приток $(\varepsilon)$ рассчитывается как производное от разности восходящих $(G \uparrow)$ и нисходящих $(G \downarrow)$ потоков излучения на высоте $H$

$$
\varepsilon(H)=-\frac{\partial[G \uparrow(H)-G \downarrow(H)]}{\partial H}
$$

или, более грубо, для слоя $\Delta H=H_{n}-H_{n-1}$ (т. н. приток к слою, $\varepsilon_{\mathrm{c}}$ )

$$
\varepsilon_{c}=-\frac{\Delta[G \uparrow(H)-G \downarrow(H)]}{\Delta H},
$$

то относительные погрешности нередко составляют десятки и даже сотни процентов. Наглядно иллюстрирует это табл. 1. Видно, что точность эффективного излучения порядка $1 \%$ не обеспечивает надежных результатов расчета радиационных притоков тепла. Естественно, что определение их подробного вертикального профиля (по формуле (1)) требует еще более точной и обстоятельной информации о величинах $G \uparrow-G \downarrow$. Решение этой проблемы не входило, однако, в задачи нашей статьи.

Как отмечалось при анализе рис. 1, одной из наиболее важных исходных характеристик для расчета противоизлучения атмосферы является общее влагосодержание выше того уровня, для которого определяются значения радиации. В этой связи интересно выяснить, насколько результаты таких расчетов чувствительны к изменениям вертикального хода влажности при неизменной величине общего содержания водяного пара. Расчеты были произведены с использованием данных всех пяти моделей, причем для каждой из них выбрано восемь вариантов вертикального распределения влажности. Результаты для модели II частично показаны на рис. 3 (выводы справедливы и для остальных моделей). 
Таблица 1

Значения радиационных притоков тепла $\left.\varepsilon_{\mathrm{c}}, 10^{-5} B T\right\rceil c M^{3}$, к слоям толщиной $1 \kappa \mathrm{k}$ и соответствующие относительные погрешности* $\left|\delta \varepsilon_{\mathrm{c}}\right|$, \%

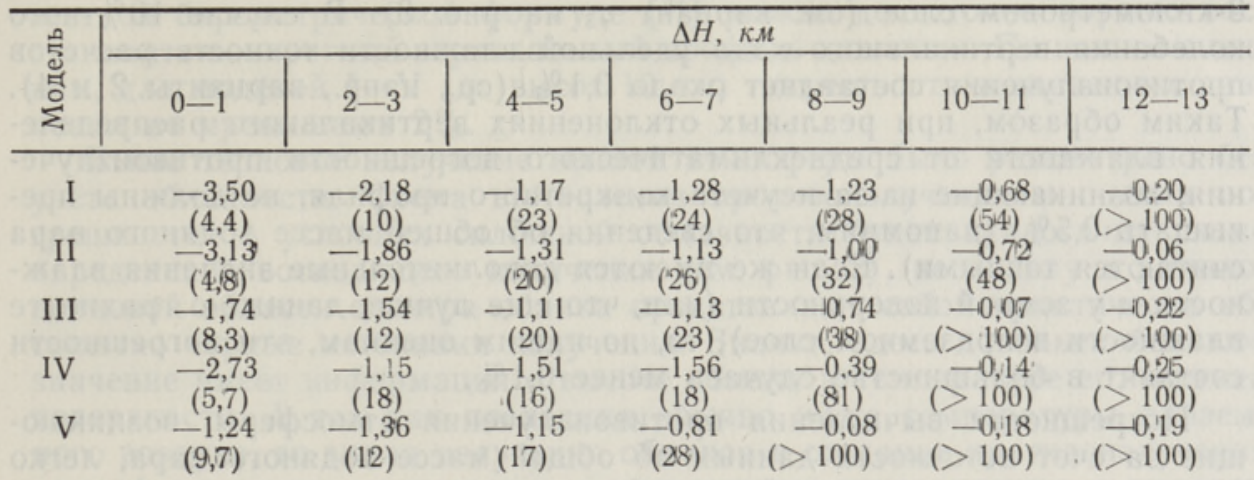

* Погрешности $\left|\delta \varepsilon_{\mathrm{c}}\right|$ (даны в скобках) определены в предполюжении, что величины $G \uparrow-G \downarrow$ получены с точностью $1 \%$.

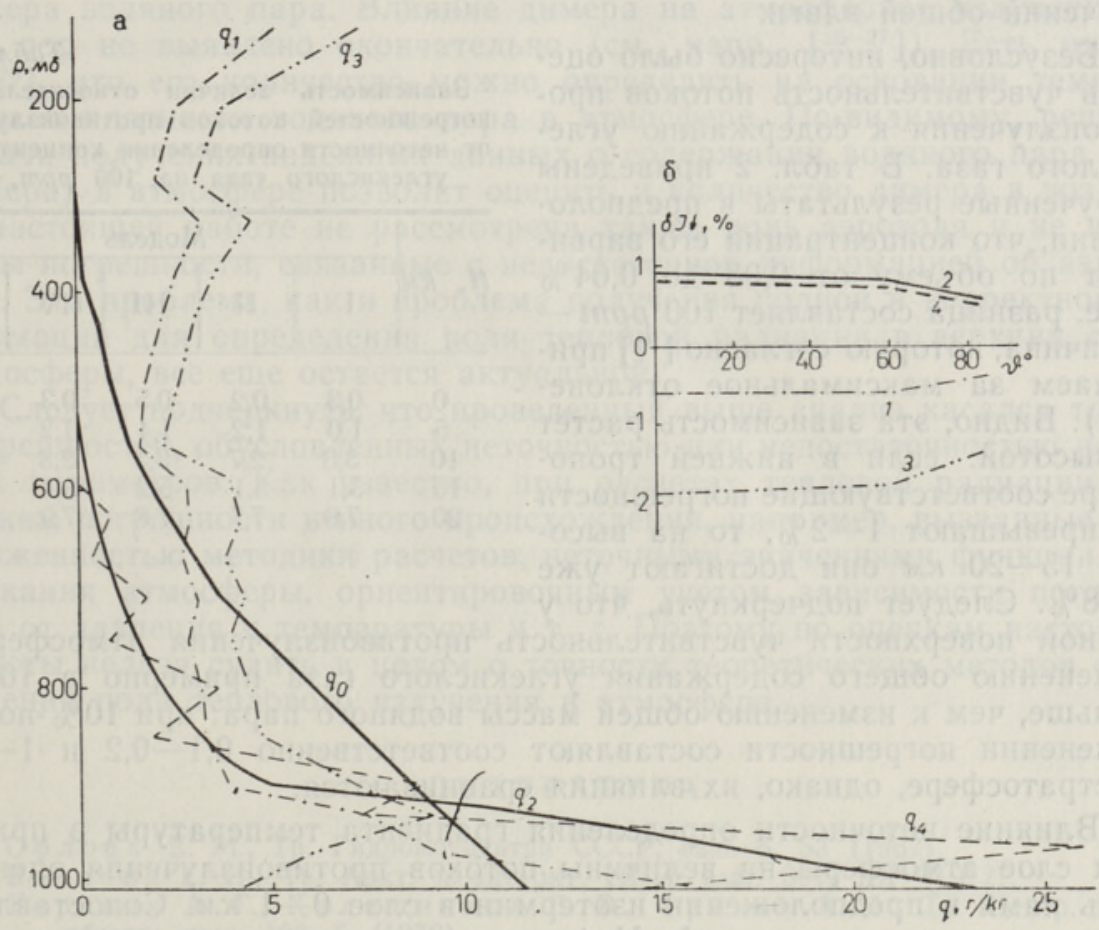

Рис. $3, a-$ Различные варианты вертикального распределения удельной влажности $(q)$ и б - соответствующие относительные погрешности интенсивности нисходящей радиации атмосферы $\quad\left(\delta I \downarrow=\frac{I_{i} \downarrow-I_{0} \downarrow}{I_{0} \downarrow}\right)$, рассчитанные относительно модели II ( $\bar{q}_{0}$ и $\left.I_{0} \downarrow\right)$.

Как видно, даже очень значительные расхождения в вертикальном ходе влажности (достигающие и $\pm 100 \%$ по сравнению с базовой моделью) не оказывают существенного влияния на результаты вычисления проти- 
воизлучения. Максимальные отклонения в значениях радиации не превышали $2 \%$, но и такие погрешности можно ожидать лишь при больших односторонних отклонениях удельной влажности во всем нижнем 3-километровом слое (см. вариант $q_{3}$ на рис. 3 ). В случае $10 \%$-ного колебания вертикального хода удельной влажности точность расчетов противоизлучения составляет около $0,1 \%$ (ср., напр., варианты 2 и 4). Таким образом, при реальных отклонениях вертикального распределения влажности от среднеклиматического погрешности противоизлучения, возникающие из-за неучета конкретного профиля, не должны превышать 0,5\% (напомним, что сведения об общей массе водяного пара считаются точными). Если же имеются дополнительные значения влажности и у земной поверхности (или, что еще лучше, данные о градиенте влажности в приземном слое), то, по нашим оценкам, эти погрешности составят в большинстве случаев менее $0,1 \%$.

Погрешности вычисления противоизлучения атмосферы, возникающие за счет неточности данных об общей массе водяного пара, легко оценить для изотермической атмосферы, где на потоки теплового излучения не влияет высотное распределение поглощающих веществ. Найдено, что $10 \%$-ная неточность влечет за собой изменение противоизлучения порядка $1-2 \%$ и что эта величина мало зависит от конкретных значений общей влаги.

Безусловно, интересно было оценить чувствительность потоков противоизлучения к содержанию углекислого газа. В табл. 2 приведены полученные результаты в предположении, что концентрация его варьирует по объему от 0,03 до $0,04 \%$ (т. е. разница составляет $100 \mathrm{ppm-}$ величина, которую согласно [ [ $\left.{ }^{23}\right]$ принимаем за максимальное отклонение). Видно, эта зависимость растет с высотой: если в нижней тропосфере соответствующие погрешности не превышают $1-2 \%$, то на высотах 15-20 км они достигают уже $5-8 \%$. Следует подчеркнуть, что у

таблица 2

Зависимость величин относительных погрешностей потоков противоизлучения от неточности определения концентрации углекислого газа на $100 \mathrm{ppm}, \%$

\begin{tabular}{r|c|c|c|c|c}
\hline \multirow{2}{*}{ H, км } & \multicolumn{5}{|c}{ Модель } \\
\cline { 3 - 6 } & I & II & III & IV & V \\
\hline 0 & 0,3 & 0,2 & 0,5 & 0,3 & 0,6 \\
5 & 1,0 & 1,2 & 1,4 & 1.2 & 1.7 \\
10 & 3,0 & 2,7 & 3,3 & 2,3 & 3,6 \\
15 & 5,1 & 5,1 & 5,2 & 5,4 & 5,8 \\
20 & 7,6 & 7,7 & 7,8 & 7,9 & 8,2
\end{tabular}

земной поверхности чувствительность противоизлучения атмосферы к изменению общего содержания углекислого газа примерно в 10 раз меньше, чем к изменению общей массы водяного пара: при 10\%-ном их изменении погрешности составляют соответственно $0,1-0,2$ и $1-2 \%$. В стратосфере, однако, их влияния сравниваются.

Влияние неточности определения градиента температуры в приземном слое атмосферы на величины потоков противоизлучения оценивались нами в предположении изотермии в слое $0-1 \kappa м$. Сопоставление с неизменными моделями I-V (вертикальные градиенты температуры в слое $0-1 \kappa м$ соответственно - $6 ;-4 ;-3,5 ;-5$ и $+2 \%$ км) дало расхождение $1,5-3,5 \%$. Таким образом, неучет градиента температуры величиной $1 \%$ км в нижнем километровом слое обусловливает погрешность противоизлучения атмосферы на земной поверхности порядка $0,6 \%$.

Для расчетов теплового излучения в атмосфере необходимо, как известно, располагать данными о вертикальном распределении давления воздуха. При наличии лишь наземных значений давления приходится либо восстанавливать вертикальный профиль давления, либо ис- 
пользовать некоторые среднеклиматические данные. Но если дополнительно имеются сведения о вертикальном градиенте температуры, то, по-видимому, предпочтение следует отдать первой возможности: по известной барометрической формуле [24] можно достаточно хорошо восстановить конкретный вертикальный профиль давления. По нашим оценкам, для моделей $\mathrm{I}-\mathrm{V}$ (до высот 10 км) погрешности в значениях давления не превышают $2 \%$.

Выше тепловое излучение земной атмосферы было рассмотрено без учета облачности. Схема вычисления противоизлучения и оценка погрешностей в условиях сплошной облачности будет аналогична схеме определения восходящего излучения (см. рис. 2), поскольку в длинноволновой области спектра облака представляют собой практически абсолютно черные источники излучения. Значит, здесь довольно большое значение имеет информация о высоте облака и температуре его нижней поверхности. А так как последняя обычно ниже температуры приземного воздуха, то вклад излучения облаков в создание противоизлучения атмосферы может оказаться заметно меньше по сравнению с вкладом излучения земли в формирование потока восходящего излучения (особенно при больших значениях влаги в подоблачном слое).

В расчетные схемы настоящей работы не входил учет излучения димера водяного пара. Влияние димера на атмосферное излучение до сих пор не выявлено окончательно (см., напр., $\left.\left[{ }^{25-27}\right]\right)$. Есть мнения $\left[{ }^{25,26}\right]$, что его количество можно определить на основании температуры и плотности водяного пара в атмосфере. По-видимому, решение задачи получения надежных данных о содержании водяного пара (мономера) в атмосфере позволит оценить и количество димера в воздухе. В настоящей работе не рассмотрена также роль аэрозоля и не выявлены погрешности, связанные с недостаточной информацией об аэрозолях. Эта проблема, как и проблема получения полной и корректной информации для определения поля тепловой радиации в верхних слоях атмосферы, все еще остается актуальной.

Следует подчеркнуть, что проведенный выше анализ касался только погрешностей, обусловленных неточностью или недостаточностью исходных параметров. Как известно, при расчетах тепловой радиации возможны погрешности и иного происхождения, например, вызванные приближенностью методики расчетов, неточными значениями функции пропускания атмосферы, ориентировочным учетом зависимости поглощения от давления и температуры и т. д. Поэтому по оценкам настоящей работы нельзя судить в целом о точности теоретических методов определения поля теплового излучения в атмосфере.

\section{Л ИТ Е Р АТ У Р А}

1. Орлов а Е. М., Тр. Гидрометцентра СССР, вып. 6, 53 (1967).

2. Б аз лов а Г. И., Тр. Аркт. и антаркт. НИИ, вып. 301,131 (1972).

3. Горелик А. Г., К ал ашников В. В., Фролов А. А., Тр. Центр. аэрол. обсерв., вып. 103, 5 (1972).

4. Дам.бков к а Я Е. П., Метеорол. и гидрология, № 8, 8 (1969).

5. Конд р а ть в К. Я., Т и мо фе е Ю. М., Термическое зондирование атмосферы со спутников, Л., 1970.

6. Sm i th, W. L., H ow e 11, H. B., J. Appl. Meteorol., 10, No. 5, 1026 (1971).

7. Тимофеев Ю. М., Покровский О. М., Кузнецов А. Д., Метеорол. и гидрология, № 3,102 (1972).

8. W a n g, I. Y., J. Atmos. Sci., 31, No. 2, 513 (1974).

9. Пле чк ов В. М., Изв. АН СССР, Физ. атмосф. и океана, 4, № 2,182 (1968).

10. Гу рв ич А. С., Д е мин В. В., Изв. АН СССР, Физ. атмосф. и океана, 6, № 8, 771 (1970).

11. Плечков В. М., Гурвич А. С., Снопков В. Г., Докл. АН СССР, 193, № 5, 1041 (1970). 
12. Rosenkranz, P. W., Barath, F. T., Blinn, J. C., Johnston, E. J., Le noir, W. B., Sta elin, D. H., W a ters, J. W., J. Geophys. Res., 77, No. 30, 5833 (1972)

13. Кондратьев К. Я., Мелентьев В. В., Рабинович Ю. И., Шульгина Е. М., Вод. ресурсы, № 2, 58 (1973).

14. Митник Л. М., Метеорол. и гидрология, № 6, 22 (1974).

15. Wark, D. Q., Lienisch, J. H., Weinreb, M. P., Appl. Optics, 13, No. 3, 507 (1974)

16. Н ийли с к Х., В сб.: Радиация в атмосфере, Тарту, 1969, с. 60.

17. M c Cl a t c h ey, R. A., F e n n, R. W., S elby, J. E. A., V olz, F. E., G a r in g, J. S., Optical Properties of the Atmosphere. Air Force Cambridge Research Laboratories. Environmental Research Papers No. 411, August 1972.

18. К онд р а ть в К. Я., Актинометрия, Л., 1965.

19. D e a c o n, E. L., Quart. J. R. Meteorol. Soc., 96, No. 408 (1970).

20. S w in b a n k, W. C., J. Roy. Soc., 89, No. 381, 21 (1963).

21. Гидрюк Г. В., Малевский-М алевич С. П., Тр. ГГО, вып. 297, 124 (1973).

22. Re ber, E. E., Sw op e, J. R., J. Appl. Meteorol., 11, No. 8, 1322 (1972).

23. Радиационные характеристики атмосферы и земной поверхности, под ред. К. Я. Конд р а т ев а, Л., 1969.

24. Т в ерско й П. Н., Курс метеорологии, Л., 1962.

25. Викторов а А. А.; Жев акин С. А., Докл. АН СССР, 194, № 2, 291 (1970).

26. По бе р ов ск и й А. В., В сб.: Проблемы физики атмосферы, вып. 11, 1974, с. 46.

27. Д и анов-Клоков В. И., Иванов В. М., Савицкий Ю. А., Изв. АН СССР, Физ. атмосф. и океана, 11, № 12, 1247 (1975).

Ннститут термофизики и электрофизики Академии наук Эстонской ССР

Поступила в редакцию 7/VI 1976

Helgi ARST

\section{MAA ATMOSFÄARI SOOJUSKIIRGUSE ARVUTAMISEST MITTETÄIELIKE LÄHTEANDMETE KORRAL}

Artiklis käsitletakse mitmesuguste lähteparameetrite mōju atmosfääri soojuskiirguse voogude arvutamise tulemustele. Hinnatakse infrapunaste kiirgusvoogude ja voo divergentsi väärtuste võimalikke ebatäpsusi, mida põhjustab meteoroloogiliste algandmete puudulikkus. Analüüsitakse võimalusi kasutada arvutustes keskmisi klimatoloogilisi andmeid ja olemasolevate meteoroloogiliste andmete ekstrapolatsiooni.

Helgi ARST

\section{ON THE CALCULATIONS OF THERMAL RADIATION IN THE EARTH'S ATMOSPHERE IN CASE OF INCOMPLETE INITIAL DATA}

The influence of several initial parameters on the results of calculations of thermal radiation in the Earth's atmosphere is considered. The errors of the values of infrared radiation fluxes and flux divergence, caused by errors or by the incompleteness of the initial meteorological data, are estimated. The author analyses the possibilities of applying in the calculations the averaged ciimatic data and an extrapolation of the actual meteorological data. 